$\angle$ Research Square
Preprints are preliminary reports that have not undergone peer review.

They should not be considered conclusive, used to inform clinical practice, or referenced by the media as validated information.

\title{
Two Year Progression of Working Memory Impairments In Early Relapsing- Remitting Multiple Sclerosis: Appraisal of Working Memory Profiles And Measurement Sensitivity
}

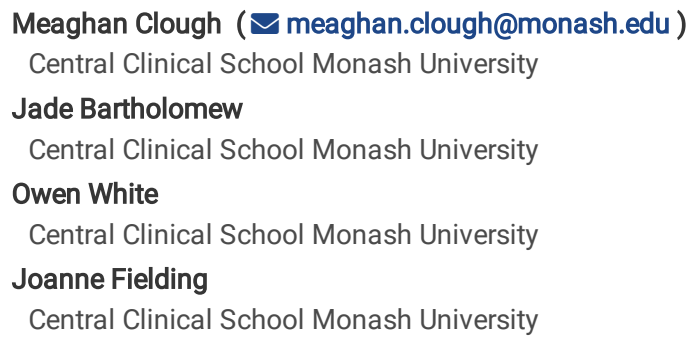

Keywords: Multiple sclerosis, working memory, working memory assessment, cognition, ocular motor, early multiple sclerosis

Posted Date: June 15th, 2021

DOI: https://doi.org/10.21203/rs.3.rs-590272/v1

License: () (1) This work is licensed under a Creative Commons Attribution 4.0 International License. Read Full License 


\section{Abstract}

Working memory (WMem) impairments are a devastating symptom of Multiple Sclerosis (MS) that manifest as distinct profiles depending upon the type of impairment. It is unknown what WMem impairment profiles occur in early MS and how best to diagnose and measure their progression.

88 participants (63 early relapsing-remitting MS, 25 healthy controls) were reviewed annually for two years (baseline, +1 year, +2 years) and completed five WMem tasks: oculomotor (OM) $n$-back-visual spatial sketchpad; digit-span forwards-phonological loop; California Verbal Learning Test (CVLT)-episodic buffer; digit-span backwards- central executive; symbol digit modalities test (SDMT)-cognitive processing speed.

$65 \%$ of RRMS patients exhibited visual-spatial WMem impairments followed by $32 \%$ for episodic, $30 \%$ phonological loop, $24 \%$ central executive, $11 \%$ cognitive processing speed. These manifested alone (51\%) or in combination (48\%), with combined impairments the best marker of general WMem impairment. Significant progression in visual-spatial impairments was found for $24 \%$ of RRMS patients, with the OM $n$-back maintaining sensitivity to general WMem impairment and progression. No other WMem subcomponent progressed or task maintained sensitivity.

The results of this study provides crucial knowledge for the creation of interventions to treat WMem impairment in early RRMS, providing key targets for treatment and endpoints for determining efficacy.

\section{Introduction}

Multiple Sclerosis (MS) is a chronic disease of the central nervous system, characterised by an interplay between neurodegenerative and inflammatory processes. Cognitive impairment is reported in up to $70 \%$ of people with MS (pwMS) irrespective of disease subtype ${ }^{1}$. Changes to working memory (WMem) are common, affecting the proficiency with which pwMS maintain and manipulate different types of information in an "active" and accessible format ${ }^{2-4}$; this in turn impacts higher cognitive processes associated with goal directed behaviour ${ }^{5}$. In recent years a focus on developing interventions/rehabilitation strategies has occurred to varying success ${ }^{6}$, with success seemingly maximised when the specific impairment is targeted, appropriate measures are selected ${ }^{7}$, and intervention occurs early when neural compensatory mechanism are still active ${ }^{8}$. At present it is unknown how WMem impairments manifest and progress in early MS (WMem profiles), and which measures are best for diagnosing impairment and detecting change.

A prominent WMem theory by Baddeley and Hitch ${ }^{9,10}$, posits that WMem consists of four specialised subcomponents that form a widely dispersed functionally integrated, yet partially segregated (according to subcomponent) neural network ${ }^{11}$. The phonological loop and visuospatial sketchpad subcomponents, actively (rehearsal) and passively maintain auditory and visual spatial information, respectively. The episodic buffer subcomponent represents a storage system that integrates newly acquired information from the visual-spatial sketchpad and/or phonological loop with long-term memory. Lastly, a central executive subcomponent coordinates the aforementioned subcomponents, controlling the focus of attention in order to maximise the proficiency of information maintenance and integration with other cognitive processes $9,10,12$. The capacity of each subcomponent is considered variable both within and between individuals, a consequence of modifying factors such as genetics, age, gender, intelligence, mood, and, in the case of disease, disease specific factors such as disease duration and severity ${ }^{13-15}$. Given, the widespread nature of MS pathology and the heterogeneity observed between pwMS, it is likely that individual differences in which WMem subcomponent(s) is impaired as well as the combination of subcomponents impaired occurs.

WMem impairments have been reported in pwMS within all subcomponents, although they are most frequently associated with the episodic buffer ${ }^{1,16-19}$. However, in early MS, impairments appear to primarily involve the visual spatial sketchpad and phonological loop, with some reports to suggest a sparing of the executive and episodic buffer ${ }^{20,21}$. This also appears to be the case for those with a clinically isolated syndrome ${ }^{15,21-23}$, which is the first clinical manifestation of MS in approximately $85 \%$ of patients ${ }^{20}$. To our knowledge only a single study has examined different WMem subcomponents impairments within a single study in pwMS with significant disability (EDSS $\sim 4)$ and advanced disease duration ( 10 years). Results of this study are largely in line with the aforementioned findings in pwMS, with impairments found for all subcomponents but most severely for the episodic buffer. However, it is unclear from this work, the frequency with which subcomponent impairments co-occur within patients, whether impairments are present in patients with early disease, and whether impairments differentially progress once disease modifying and individual factors are considered.

The aim of this study was to assess the different WMem subcomponents in pwMS with early disease (EDSS $\leq 2)$ to determine (1) the frequency of impairment within different WMem subcomponents, (2) progression of each subcomponent, and (3) which measures offer the best biomarkers in terms of diagnostic accuracy and as a measure of progression. It was anticipated that pwMS would exhibit a greater frequency of impairments within the visual spatial sketchpad and phonological loop relative to controls. Further, that WMem subcomponents would progress at different rates over the study period, with certain measures offering sensitive biomarkers of impairment.

\section{Methods}

\section{Participants}

Sixty-three patients, 14 clinically isolated syndrome (CIS), 49 with clinically definite MS (CDMS) diagnosed as early relapsing-remitting MS (RRMS, EDSS $\leq 2$ ), were included. All CDMS patients were diagnosed based on the McDonald criteria 2017 revision, all CIS patients had an initial neurological event with MRI abnormality consistent with demyelination. No patient had experienced a clinical event within 60 days prior to testing. Twenty-five healthy control participants without a history of neurological or psychiatric illness, and normal visual acuity were recruited. Further details can be found in table 1 . All participants gave their informed consent prior to completing the study. All ethical procedures complied with the ethical standards of the relevant institutional committees 
(Melbourne Health Human Research Ethics Committee, 2007.094; Monash University Human Research Ethics Committee, 2017.8068) and the Helsinki Declaration of 1964 and its later amendments.

\section{Study design}

This was a prospective, longitudinal study conducted over a 2 year observational period; baseline, +1 year, +2 years. Average times between visits were baseline to +1 year $(M=11.35$ months $S D=1.73),+1$ year to +2 years $(M=11.21$ months $S D=1.72)$. Total attrition across the study period was $14.29 \%(n=$ 9).

\section{Clinical measures: Modifying factors}

Measures of depression (Beck Depression Inventory, BDI), premorbid intelligence (National Adult Reading Test, NART), disability (EDSS) and disease duration (months since first symptom) were collected at each study visit (intelligence at baseline only) and administered according to standardised instructions (see below for details). For all except intelligence, higher values indicated worse performance/severity.

\section{Tests of WMem}

The following tests were administered according to standardised instructions at each study visit (baseline, +1 year, +2 years). For the ocular motor $n$-back task, set up and administration was conducted in accordance with the published methodology as outlined in ${ }^{20}$

\section{Episodic buffer: California Verbal Learning Test (CVLT-II)}

The CVLT is a comprehensive measure of verbal memory, with the immediate recall section providing a measure of the episodic buffer subcomponent of WMem ${ }^{16,18,24}$.

Phonological loop: digit span forwards

Digit span forwards requires reciting a number sequence in the same order as verbally presented, and provides a measure of the phonological loop ${ }^{18,25}$.

\section{Central executive: digit span backwards}

Digit span backwards requires reciting a number sequence in the reverse order as verbally presented and provides a measure of the central executive ${ }^{18,25}$.

Visual spatial sketchpad: ocular-motor n-back task

The ocular motor n-back task provides a measure of the visual spatial sketchpad, and requires participants to retain visual and spatial information in order to inform an eye movement response ${ }^{20,26}$. Here, we present data that reflect two WMem loads, 0-back, and 1-back, respectively. The measures of interest were response time of a correct response, and error. Response time (ms) was calculated as the temporal difference between fixation offset and saccade onset using a velocity criterion of $30^{\circ}$ per second, where saccades were initiated $>100 \mathrm{~ms}$ post cue disappearance and ended within $2 \cdot 5^{\circ}$ of the centre of the correct box. An error was defined as a saccade to an incorrect spatial location, or where no attempt to respond was made. Error rate was calculated for each WMem load (0-back, 1-back) as a percentage of total trials.

\section{Cognitive processing speed: symbol digit modalities test (SDMT).}

The SDMT is a measure of attention, processing speed and spatial WMem, and is considered the gold standard measure of cognitive processing speed in pwMS $^{27}$.

\section{Data analysis}

IBM SPSS statistics package version 24 was used for all statistical analyses. Baseline differences between groups in EDSS, disease duration (months since first symptom), depression, intelligence, and age, were determined by t-tests. Pearson's chi-square analysis was performed to determine difference in sex and frequency of WMem subcomponent impairments across groups. Z-scores were calculated to determine subcomponent impairment, where controls

represented the normative population: $Z=\frac{x-\mu}{\delta}$, where $x=$ observed value, $\mu=$ mean of normative sample, $\sigma=$ standard deviation of the normative sample. For the CVLT/SDMT/digit spans a $z$-score of $\leq-1.5$ indicated failure; a z-score of $\geq 1.5$ indicated failure on OM $n$-back.

Diagnostic accuracy for WMem measure was determined for their capacity (at baseline and +2 years) to discriminate, (1) pwMS from healthy controls, and (2) individuals with a single subcomponent WMem impairment and (3) a multi-subcomponent WMem impairment ( $\geq$ two WMem subcomponents impaired). Area under the curve (AUC) values found to be significantly above .5 indicated diagnostic accuracy significantly greater than chance.

Change in WMem performance over time for each WMem measure was determined using a mixed linear regression adjusted model. For all models, factors included time (baseline, + 1 year, and +2 years) and group (CIS, CDMS). For the OM n-back an additional factor of WMem load (0-back, 1 back) was included. Fixed effects and interactions were generated for each. A subject-specific random effect was included in all models to account for between-subject heterogeneity. Covariates explored were age, intelligence (NART), depressive symptomology (BDI), disease duration (months since first symptom(s)), and sex (male, female). Where theoretically relevant effects/interaction were deemed relevant covariates, these were included as fixed effects. All models were based on 344 data points for OM $n$-back and 174 data points for the CVLT, SDMT and digit span. 
Reliable change indices (RCls) were calculated to determine the proportion of pwMS whose performance had significantly deteriorated $(p<.05)$ between baseline and +2 years. Calculation of RCls was conducted in accordance with the Jacobson-Truax index procedure $\frac{x_{1-x_{2}}}{s_{\text {diff }}}, \mathrm{x}_{1}=$ baseline WMem performance, $x_{2}=+2$ years WMem performance, $S_{\text {diff }}=\sqrt{2\left(S_{E}\right)^{2}}$ and $S_{E}=$ standard error of measurement. For the CVLT/SDMT/digit spans a RCl score of $\leq$ -1.5 indicated significant $(p<.05)$ deterioration in performance from baseline; $z$-score of $\geq 1.5$ was used for OM $n$-back.

\section{Results}

\section{Descriptive information for pwMS and healthy controls}

CIS and CDMS groups were not significantly different from healthy controls in terms of age, depressive symptomology (BDI), or premorbid intelligence (NART). Overall, CIS patients were significantly younger than CDMS patients $(F(1,63)=7.54, p=.008)$ and had a significantly shorter disease duration $(F(1,44.96)=$ 28.67, $p<.000)$; EDSS was comparable between CIS and CDMS groups. All participants exhibited visual acuity within the normal range, either corrected or uncorrected (6/4-6/6). Table 1.

Table 1. Demographic and clinical information for pwMS and healthy controls

\begin{tabular}{|c|c|c|c|c|c|c|c|c|}
\hline & \multirow{2}{*}{\multicolumn{2}{|c|}{$\begin{array}{l}\text { Healthy controls } \\
(n=25)\end{array}$}} & \multicolumn{4}{|c|}{ Early MS } & \multirow{2}{*}{\multicolumn{2}{|c|}{$\begin{array}{l}\text { Total PwMS } \\
(n=63)\end{array}$}} \\
\hline & & & \multicolumn{2}{|c|}{ CIS $(n=14)$} & \multicolumn{2}{|c|}{ CDMS $(n=49)$} & & \\
\hline & $M(\mathrm{SD})$ & Range & $M(\mathrm{SD})$ & Range & $M(\mathrm{SD})$ & Range & $M(\mathrm{SD})$ & Range \\
\hline \multirow[t]{2}{*}{ Age (years) } & 38.63 & $21-65$ & $33.00 *$ & $20-46$ & $42.18^{*}$ & $19-66$ & $40.14(11.60)$ & $19-66$ \\
\hline & (11.07) & & (8.38) & & (11.64) & & & \\
\hline Gender & $21(3)$ & - & $12(2)$ & - & $45(4)$ & - & $57(6)$ & - \\
\hline \multicolumn{9}{|l|}{$\mathrm{F}(\mathrm{M})$} \\
\hline \multirow[t]{2}{*}{ NART } & 117.00 & $110-124$ & 115.29 & $105-123$ & 115.92 & $106-125$ & 115.78 & $105-125$ \\
\hline & $(4.23)$ & & $(5.14)$ & & $(4.56)$ & & $(4.65)$ & \\
\hline \multirow[t]{2}{*}{ BDI } & 3.95 & $0-14$ & 6.85 & $1-18$ & 6.67 & $0-38$ & 6.71 & $0-38$ \\
\hline & (3.29) & & $(5.36)$ & & (8.19) & & $(7.61)$ & \\
\hline \multirow[t]{2}{*}{ EDSS } & & & 0.00 & $0-2$ & 0.00 & $0-3.5$ & 0.00 & $0-3.5$ \\
\hline & & & $(.00)$ & & $(1.00)$ & & $(1.00)$ & \\
\hline \multirow[t]{2}{*}{ Disease duration (months) } & - & - & $12.85^{\star \star}$ & $2-37$ & 104.54 ** & $4-513$ & 83.43 & $2-513$ \\
\hline & & & (11.72) & & $(110.75)$ & & $(104.54)$ & \\
\hline
\end{tabular}

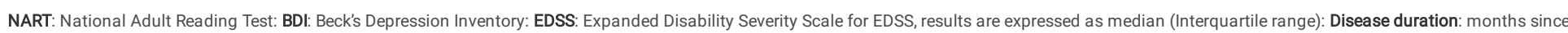
first symptom(s). Significantly different between MS subgroups at * $p<.05$ or $* \star p<.001$ significance

\section{Frequency of WMem subcomponent impairments at baseline}

Mean and standard deviations for each WMem are presented in Table 2.

Table 2. Baseline WMem subcomponent performance for controls and early MS patients. 


\begin{tabular}{|c|c|c|c|c|c|c|c|c|}
\hline & \multirow{2}{*}{\multicolumn{2}{|c|}{$\begin{array}{l}\text { Control } \\
(n=25)\end{array}$}} & \multicolumn{4}{|l|}{ Early RRMS } & \multirow{2}{*}{\multicolumn{2}{|c|}{$\begin{array}{l}\text { Total RRMS } \\
(n=63)\end{array}$}} \\
\hline & & & CIS $(n=14)$ & & CDMS $(n=49)$ & & & \\
\hline & $M(\mathrm{SD})$ & $\begin{array}{l}\text { Failed } \\
n(\%)\end{array}$ & $M(\mathrm{SD})$ & $\begin{array}{l}\text { Failed } \\
n(\%)\end{array}$ & $M(\mathrm{SD})$ & $\begin{array}{l}\text { Failed } \\
n(\%)\end{array}$ & $M(\mathrm{SD})$ & $\begin{array}{l}\text { Failed } n \\
(\%)\end{array}$ \\
\hline CVLT & $44.33(9.68)$ & $1(4.0)$ & $43.21(14.86)$ & $3(21.42)$ & $41.75(12.51)$ & $9(18.37)$ & $42.08(12.84)$ & 12(19.05) \\
\hline \multicolumn{9}{|l|}{ Episodic buffer } \\
\hline SDMT & $68.50(11.77)$ & $0(0)$ & $61.78(7.08)$ & $0(0)$ & $63.23(12.57)$ & $4(8.16)$ & $62.90(11.53)$ & $4(6.35)$ \\
\hline \multicolumn{9}{|l|}{$\begin{array}{l}\text { Cognitive processing } \\
\text { speed }\end{array}$} \\
\hline Digit span-Forwards & $12.04(2.0)$ & $1(4.0)$ & $10.71(2.09)$ & $2(14.28)$ & $10.93(2.30)$ & $9(18.37)$ & $10.88(2.24)$ & 11(17.46) \\
\hline \multicolumn{9}{|l|}{ Phonological loop } \\
\hline Digit span -Backwards & $7.75(2.23)$ & $0(0)$ & $6.78(1.67)$ & $0(0)$ & $6.78(1.67)$ & $9(18.37)$ & $6.81(2.11)$ & $9(14.28)$ \\
\hline
\end{tabular}

Central executive

\section{OM n-back}

Visual-spatial sketchpad

\begin{tabular}{|c|c|c|c|c|c|c|c|c|}
\hline O-back response time & $\begin{array}{l}582.76 \\
(119.31)\end{array}$ & $0(0)$ & $\begin{array}{l}535.10 \\
(114.43)\end{array}$ & $0(0)$ & $\begin{array}{l}596.01 \\
(139.98)\end{array}$ & $5(10.20)$ & $582.46(136.26)$ & $5(7.94)$ \\
\hline 1-back response time & $\begin{array}{l}587.85 \\
(135.01)\end{array}$ & $0(0)$ & $\begin{array}{l}544.50 \\
(114.15)\end{array}$ & $0(0)$ & $\begin{array}{l}604.79 \\
(175.85)\end{array}$ & $5(10.20)$ & $591.39(165.26)$ & $5(7.94)$ \\
\hline O-back error & $7.86(11.40)$ & $4(16.0)$ & $12.46(9.98)$ & $1(7.14)$ & $20.62(22.80)$ & $14(28.57)$ & 18.80(20.86) & $15(23.81)$ \\
\hline \multirow[t]{2}{*}{ 1-back error } & $14.21(12.80)$ & (0) & $21.84(13.93)$ & $3(2.14)$ & $29.91(23.96)$ & $17(34.69)$ & 28.11(22.29) & $20(31.75)$ \\
\hline & Total failed $(n)$ & Failed (\%) & Total failed $(n)$ & Failed (\%) & Total failed $(n)$ & Failed (\%) & Total failed $(n)$ & Failed (\%) \\
\hline Failed 1 WMem measure & 6 & 20.0 & 3 & 24.43 & 14 & 28.5 & 17 & 26.98 \\
\hline $\begin{array}{l}\text { Failed } \geq 2 \text { WMem } \\
\text { measures }\end{array}$ & 0 & 0 & 3 & 21.43 & 17 & 32.65 & 20 & 31.75 \\
\hline Total failed & 6 & 20.0 & 6 & 42.86 & 31 & 63.27 & 37 & 58.73 \\
\hline
\end{tabular}

CVIT: California Verbal Learning Test II: SDMT: Symbol Digit Modalities Test: OM: Ocular Motor

PWMS were significantly more likely to have an impairment in at least 1 WMem subcomponent $\left(X^{2}(1, N=88)=9.20, p=.002\right)$ and a multi sub-component WMem impairment $\left(X^{2}(1, N=88)=9.26, p=.002\right)$ than healthy controls.

The majority of pwMS with a WMem impairment had an impairment in the visual-spatial sketchpad (64.86\%), followed by the episodic buffer (32.43\%), phonological loop (29.73\%), central executive (24.32\%), and cognitive processing speed (10.81\%). See Fig. 1.

The majority of WMem impaired pwMS had a single WMem subcomponent impairment (51.37\%), the most common being an isolated visual-spatial sketchpad impairment (21\%), or an isolated episodic buffer impairment (16\%). Further, $40.54 \%$ of impaired pwMS had a WMem impairment that included two subcomponents, the two most common being visual-spatial sketchpad + episodic buffer (11\%), or visual-spatial sketchpad + central executive (11\%) impairment. Only $9 \%$ of patients had a WMem impairment that included three subcomponents. See Fig. 2.

\section{Diagnostic accuracy of WMem measures at baseline}

Diagnostic accuracy for each WMem subcomponent measure was determined for their capacity to discriminate, (1) pwMS from healthy controls, (2) pwMS with at least 1 WMem impairment from those without, and (3) pwMS with a multi-subcomponent WMem impairment (impairment in $\geq 2$ different WMem subcomponents) for those without.

The OM $n$-back and digit span forwards demonstrated diagnostic accuracy (significantly greater than chance) for discriminating pwMS from healthy controls (OM n-back: 1-back error: $\mathrm{AUC}=.69 ; 0$ - back error: $\mathrm{AUC}=.68$; digit span forwards: $\mathrm{AUC}=.65$ ).

For discriminating pwMS with a WMem impairment from those without, the OM $n$-back (1-back error: AUC $=.70 ; 0-$ back error: AUC $=.64$ ), digit span forwards $(A U C=.68)$ and CVLT $(A U C=.67)$ demonstrated significant diagnostic accuracy.

In contrast, all WMem measures except for the SDMT and CVLT demonstrated diagnostic accuracy significantly greater than chance for discriminating pwMS with multi-subcomponent WMem impairment from those without (OM $n$-back: 1 -back error: AUC = $.83 ; 0-$ back error: $A U C=.72$; digit span forwards: AUC $=.78$; digit span backwards: AUC $=.74)$.

Change in WMem subcomponent performance over two years (baseline, +1 year, +2 years) 
All adjusted means and standard deviations for each WMem measure and clinical covariates at each study visit can be found in Table 3 .

Visual-spatial sketchpad: OM n-back

Error

A significant effect of time was found $(F(2,144.90)=3.41, p=.036)$, with error rate significantly reducing on average by $6.70 \%(S E=3.4)$ across the three study visits: $5.10 \%(\mathrm{SE}=2.89)$ between baseline and +1 year, $.17 \%(\mathrm{SE}=2.62)$ between +1 year and +2 years. A time by group interaction $(F(2,143.18)=3.45, p$ $=.034)$ revealed that the significant reduction in error was only present for the CDMS group $(d f(2,122.38)=11.79, p=.000021$ : baseline -+1 year mean difference $=13.35(\mathrm{SE}=.42), d f=164.63, p=.000015,95 \% \mathrm{Cl}: 6.52,20.19$; baseline -+2 years mean difference $=10.65(\mathrm{SE}=.44), d f=135.04, p=.0001,95 \%$ Cl: 3.83-17.47). Two pwMS (1 CIS, 1 CDMS) showed significant worsening in error rate.

\section{Response time}

In addition to the standard covariates, error*RRMS subgroup *time was included to account for the possibility of a speed/accuracy trade-off; the significant reduction in error rate over time raises the possibility that the increase in response time may be a consequence of patients sacrificing speed (prolonging response time) to increase accuracy (reducing error rate). A significant effect of time was evident $\left(F(2,141.32)=22.73, p=2.76 \times 10^{-9}\right)$, with response time found to increase on average $188.89 \mathrm{~ms}(\mathrm{SE}=29.10)$ across the three study visits; $122.68 \mathrm{~ms}(\mathrm{SE}=24.59)$ between baseline and +1 year, $66.21 \mathrm{~ms}(\mathrm{SE}=$ 23.37) between +1 year and +2 years. A significant subgroup by time interaction was also evident $(F(2,138.18)=5.59, p=.005)$, with both CIS (average mean difference $=291.77(\mathrm{SE}=39.89), d f(2,149.49)=14.84, p=.000001)$ and CDMS ( average mean difference $=86.01(\mathrm{SE}=5.26), d f(2,128.03)=8.14, p=.00047)$ demonstrating a significant increase in response time; only the CIS group demonstrated a significant increase between each consecutive time point (baseline +1 year mean difference $=-161.21(\mathrm{SE}=42.87), d f=169.72, p=.001,95 \% \mathrm{Cl}:-264.87,-57.56 ;+1$ year -+2 years mean difference $=-130.56(\mathrm{SE}=42.54), d f=$ $106.65, p=.008,95 \% \mathrm{Cl}:-234.07,-27.04)$, while the CDMS group only demonstrated a significant increase between baseline and +1 year (mean difference $=$ $-84.16(\mathrm{SE}=24.59), d f=156.22, p=.002,95 \% \mathrm{Cl}$ - $-143.67--24.64)$. In total, 15 pwMS $(23.81 \%$ : $5 \mathrm{CIS}, 10 \mathrm{CDMS})$ showed significant slowing of response time.

Table 3. Adjusted averages for WMem measures and clinical covariates across the study visits

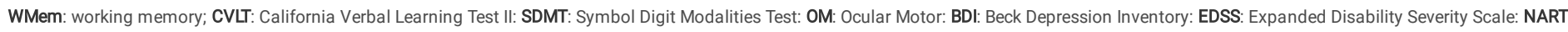
National Adult Reading Test (Pre-morbid intelligence). + NART was administered once at baseline with same scored used as a covariate at each study visit

\section{Episodic WMem: CVLT}

A significant effect of RRMS subgroup was evident $(F(1,91.11)=7.48, p=.007)$ with the CDMS group performing poorer than the CIS group (mean difference $=43.37(\mathrm{SE}=14.46), d f(1,95.06)=9.02, p=.003,95 \% \mathrm{Cl}: 14.50,72.05)$. A significant RRMS subgroup by time interaction was found $(F(2,57.79)=7.43, p$

$=.001)$, with the CDMS subgroup alone demonstrating an average of $6.67(2.64)$ point improvement over time $(d f(2,71.86)=3.62, p=.032)$; significant improvement occurred between baseline and +2 years only $(d f=63.94, p=.037,95 \% \mathrm{Cl}: 13.21, .31)$. Only one pwMS (CDMS) showed significant reduction in performance.

\section{Phonological loop: Digit span forwards}

No significant effects or interactions were evident. In total 3 pwMS (CDMS) showed significant reduction in performance.

Change in central executive: Digit span (backwards)

No significant effects or interactions were evident. In total 2 patients (CDMS) showed significant reduction in performance.

\section{Cognitive processing speed: SDMT}

A significant effect of RRMS subgroup was found $F(1,88.10)=6.01, p=.016)$, however, no significant difference between RRMS subgroups were evident. No other significant effects or interactions were evident. No individual patient was found to have a significant reduction in performance across the study period.

\section{Diagnostic accuracy of each WMem biomarker after repeated administrations}

Only the OM $n$-back maintained diagnostic accuracy for discriminating pwMS from healthy controls ( 1 -back response time: AUC $=.71 ; 0-$ back response time: $A U C=.70 ; 0$-back error: AUC = .65) and for discriminating pwMS with multi-subcomponent WMem impairment from those without $(7$-back error: $A U C=.70)$.

\section{Discussion}

The aim of this study was to determine (1) the type(s) of WMem impairments that most frequently occur in early RRMS and whether these more commonly occur as single or multi-subcomponent impairments, (2) how each of these subcomponents progresses over time, accounting for individual and disease modifying factors, and (3) what biomarkers provide the best measure of WMem impairment both as a one off assessment and following repeated administration.

Frequency of WMem subcomponent impairment in early RRMS

WMem impairment was found in over $50 \%$ of this early RRMS cohort, with the majority being visual-spatial sketchpad impairments (64.86\%), followed by 


$\begin{array}{llllllll}\text { Baseline } & +1 \text { year } & +2 \text { year } & \text { Baseline } & +1 \text { year } & +2 \text { year } & \text { Baseline } & +1 \text { year } \\ n=14 & n=14 & n=12 & n=49 & n=45 & n=42 & n=63 & n=59\end{array}$

\begin{tabular}{|c|c|c|c|c|c|c|c|c|c|}
\hline \multicolumn{10}{|l|}{$\begin{array}{l}\text { WMem } \\
\text { measures }\end{array}$} \\
\hline CVLT & 89.20 & 79.72 & 80.63 & 40.12 & 42.38 & 46.97 & 64.66 & 61.05 & 63.80 \\
\hline $\begin{array}{l}\text { Episodic } \\
\text { buffer }\end{array}$ & (15.75) & (14.28) & (12.25) & $(1.88)$ & $(2.46)$ & (1.91) & (7.93) & (7.24) & $(6.21)$ \\
\hline SDMT & 61.64 & 67.25 & 71.93 & 64.04 & 66.91 & 67.59 & 62.84 & 67.08 & 69.76 \\
\hline $\begin{array}{l}\text { Cognitive } \\
\text { processing } \\
\text { speed }\end{array}$ & (14.63) & (12.84) & (12.09) & $(1.71)$ & $(1.90)$ & $(2.81)$ & (7.37) & (6.49) & $(6.21)$ \\
\hline $\begin{array}{l}\text { Digit span- } \\
\text { Forwards }\end{array}$ & 15.65 & 15.60 & 16.90 & 11.19 & 11.17 & 11.36 & 13.42 & 13.39 & 14.13 \\
\hline $\begin{array}{l}\text { Phonological } \\
\text { loop }\end{array}$ & $(2.84)$ & $(2.50)$ & $(2.10)$ & $(.34)$ & $(.41)$ & $(.36)$ & $(1.43)$ & (1.27) & (1.07) \\
\hline $\begin{array}{l}\text { Digit span- } \\
\text { Backwards }\end{array}$ & 10.21 & 9.57 & 10.44 & 6.97 & 7.59 & 7.67 & 8.59 & 8.58 & 9.05 \\
\hline $\begin{array}{l}\text { Central } \\
\text { executive }\end{array}$ & $(2.67)$ & $(2.33)$ & (1.97) & $(.34)$ & $(.36)$ & (.33) & (1.34) & (1.18) & $(1.00)$ \\
\hline
\end{tabular}

\section{OM n-back}

Visual-

spatial

sketchpad

\begin{tabular}{|c|c|c|c|c|c|c|c|c|c|}
\hline $\begin{array}{l}\text { O-back } \\
\text { response } \\
\text { time }\end{array}$ & $\begin{array}{l}100.22 \\
(114.36)\end{array}$ & $\begin{array}{l}253.59 \\
(96.43)\end{array}$ & $\begin{array}{l}370.94 \\
(86.03)\end{array}$ & $\begin{array}{l}595.47 \\
(22.41)\end{array}$ & $\begin{array}{l}663.44 \\
(23.73)\end{array}$ & $\begin{array}{l}667.79 \\
(20.40)\end{array}$ & $\begin{array}{l}347.84 \\
(58.26)\end{array}$ & $\begin{array}{l}458.52 \\
(49.48)\end{array}$ & $\begin{array}{l}519.36 \\
(44.09)\end{array}$ \\
\hline $\begin{array}{l}\text { 1-back } \\
\text { response } \\
\text { time }\end{array}$ & $\begin{array}{l}72.87 \\
(112.73)\end{array}$ & $\begin{array}{l}241.92 \\
(95.26)\end{array}$ & $\begin{array}{l}385.69 \\
(80.22)\end{array}$ & $\begin{array}{l}591.88 \\
(28.28)\end{array}$ & $\begin{array}{l}692.21 \\
(20.67)\end{array}$ & $\begin{array}{l}691.58 \\
(16.81)\end{array}$ & $\begin{array}{l}332.38 \\
(58.14)\end{array}$ & $\begin{array}{l}467.07 \\
(48.69)\end{array}$ & $\begin{array}{l}538.63 \\
(41.09)\end{array}$ \\
\hline O-back error & $\begin{array}{l}11.13 \\
(13.19)\end{array}$ & $\begin{array}{l}14.38 \\
(10.99)\end{array}$ & $\begin{array}{l}7.15 \\
(9.13)\end{array}$ & $\begin{array}{l}20.96 \\
(3.12)\end{array}$ & $\begin{array}{l}8.41 \\
(2.69)\end{array}$ & $\begin{array}{l}9.25 \\
(1.77)\end{array}$ & $\begin{array}{l}16.05 \\
(6.78)\end{array}$ & $\begin{array}{l}11.40 \\
(5.66)\end{array}$ & $\begin{array}{l}8.20 \\
(4.66)\end{array}$ \\
\hline 1-back error & $\begin{array}{l}19.39 \\
(13.18)\end{array}$ & $\begin{array}{l}18.90 \\
(10.78)\end{array}$ & $\begin{array}{l}17.88 \\
(10.28)\end{array}$ & $\begin{array}{l}29.48 \\
(3.10)\end{array}$ & $\begin{array}{l}15.32 \\
(2.30)\end{array}$ & $\begin{array}{l}19.91 \\
(3.03)\end{array}$ & $\begin{array}{l}24.44 \\
(6.78)\end{array}$ & $\begin{array}{l}17.11 \\
(5.51)\end{array}$ & $\begin{array}{l}18.89 \\
(5.37)\end{array}$ \\
\hline
\end{tabular}

Clinical

covariates

\begin{tabular}{|c|c|c|c|c|c|c|c|c|c|}
\hline BDI & $4.8(1.23)$ & $3.00(1.22)$ & $4.4(1.08)$ & $6.29(1.79)$ & $6.19(1.32)$ & $4.48(.85)$ & 6.71(7.61) & $6.95(7.73)$ & $5.88(6.6$ \\
\hline EDSS Mdn & $0(0)$ & $.3(.20)$ & $.3(.20)$ & $.57(.27)$ & $.67(.28)$ & $.67(.28)$ & $.29(.14)$ & $.48(.24)$ & $.48(.24)$ \\
\hline $\begin{array}{l}\text { Disease } \\
\text { duration (m) }\end{array}$ & $15.4(6.64)$ & $27.00(6.52)$ & $39.80(5.88)$ & $125.90(30.99)$ & $137.43(31.01)$ & 149.28(30.99) & $83.43(104.54)$ & $95.70(106.17)$ & 114.98 \\
\hline Age & $34.8(3.73)$ & $35.80(3.73)$ & $37.00(3.73)$ & $42.48(2.30)$ & $43.43(2.70)$ & $44.38(2.70)$ & $40.14(11.60)$ & $41.64(11.34)$ & 42.94(1 \\
\hline $\operatorname{Sex} F(M)$ & $12(2)$ & $12(2)$ & $10(2)$ & $45(4)$ & $43(4)$ & $38(4)$ & $57(6)$ & $53(6)$ & $48(6)$ \\
\hline
\end{tabular}

NART $^{+}$

episodic buffer (32.43\%), phonological loop (29.53\%), central executive (24.32\%), and lastly cognitive processing speed (10.81\%). While impairments most commonly occurred as a single subcomponent impairment (51.37\%), 48.64\% presented as multi subcomponent impairment; most commonly visual-spatial sketchpad + episodic buffer, or visual-spatial sketchpad + central executive impairment. Further, pwMS were more likely to have a WMem impairment than healthy controls, both single and multi-subcomponent, with the presence of a multi-subcomponent impairment providing the highest discriminative power; no healthy control had a multi-subcomponent impairment.

These results highlight the frequency of WMem impairments in early RRMS. While there was variability in the type of WMem subcomponent impaired, the visual spatial WMem and episodic memory impairments were most consistently impaired; this is in line with prior research ${ }^{15,20-23,29,30}$. Further, the number of patients with WMem impairments as well as the number of WMem subcomponents impaired was higher in those with longer disease duration, a finding that reflects the broadening of implicated cognitive domains with increasing disease severity ${ }^{1,29-31}$. Finally, the finding that 1 in 5 of our healthy control showed impairment on one measure of WMem is important, suggesting that impairment reported in 20\% of pwMS might not represent disease related impairment 
but rather normal variation in WMem ${ }^{14}$. Potentially, the benchmark of a failure of 2 or more different WMem subcomponent measures might represent a better marker for distinguishing WMem impaired pwMS.

Change in WMem subcomponents over two years in early RRMS

A significant deterioration in performance was only evident for response time on the visual-spatial sketchpad task (OM $n$-back), with approximately $24 \%$ of pwMS exhibiting a significant increase in response time across the study period, and independent of improved error rate. All other WMem subcomponents stayed the same or significantly improved (episodic buffer - CVLT), with collectively less than $7 \%$ of pwMS found to have worsened. This disparity in findings might suggest different rates of progression for discrete WMem subcomponents, and/or differences in the sensitivity of the measures used.

The significant increase in response time on the OM $n$-back reflects a reduction in processing speed associated with the transmission of visual-spatial information to, and processing within, the visual spatial sketchpad; it does not reflect an inability to maintain and utilise visual-spatial information. Increased processing speed within the visual-spatial WMem network and the broader ocular motor network that is proposed to mediate its proficiency ${ }^{32,33}$, is frequently found in early RRMS with impairment appearing to worsen with time $20,31,34-39$. Indeed, cognitive processing speed is known to slow over time and commensurate to markers of disease progression ${ }^{19,40,41}$. In a study by Huijbregts et al., ${ }^{42}$ found that RRMS were more vulnerable on tasks that concurrently place demands on processing speed and visual-spatial working memory, such as the OM $n$-back. Interestingly, in our study, we did not see a commensurate reduction in performance on the SDMT, the proposed gold standard measure 27,43 that similarly places demands on visual WMem and processing speed ${ }^{44,45}$; indeed all pwMS identified as impaired by the SDMT were also identified by the OM $n$-back. This is in line with previous studies that have similarly found that the SDMT's ability to detect cognitive deterioration within $2-3$ years is poor ${ }^{46,47}$, with performance estimated to decrease by only .22 decrease per year ${ }^{48}$. This may represent a combination of practice effects and the sensitivity of the measure to subtle change.

Practice effects are a known issue with repeated neuropsychological assessment, and may manifest as improvement in performance or the absence of change upon repeated administration ${ }^{49-51}$. Indeed evidence in pwMS suggests that progression only becomes evident once impairment is greater than the masking effect of practice ${ }^{42,52}$. Broadly, practice effects are a consequence of the unavoidable implication of long-term memory that occurs naturally upon engagement in a task or activity. This engagement may result in long term consolidation of measure specific items due to rehearsal, general test familiarity, and/or the development and retention of memory devices such as grouping individual pieces of information into larger units (chunking) ${ }^{53}$; this device is commonly used on the CVLT (classifying related words into groups: animals, transport, vegetable, furniture) ${ }^{54}$, and digit span measures (grouping of individual numbers into large numbers) ${ }^{55}$. While the $\mathrm{OM} n$-back task was not free from practice effects (i.e. improvement in error rate was seen), this did not appear to impact the detection of response time slowing. OM assessment is performed using high powered cameras (sample rate of $500 \mathrm{~Hz}$ ) that necessarily afford the precision to detect and delineate subtle yet significant incremental increases in response time. Indeed previous work published by our group has demonstrated that OM assessment consistently detects impairments in early RRMS that are not evident using common neuropsychological measures of cognitive processing speed used in $\mathrm{MS}^{20,35-37,56,57}$. Further, and unlike the other working measures used in this study, OM assessment employs a multiple repeated trial design, likely contributing to the high test-retest reliability found in other studies using healthy populations ${ }^{58-60}$. In combination these psychometric properties likely mitigate the impact of practice effects on OM $n$-back response times, allowing for the detection of significant progression. Diagnostic accuracy of WMem biomarkers: baseline compared to repeated administrations

At baseline, all measures with the exception of the SDMT, demonstrated similar diagnostic accuracy for distinguishing a pwMS with a WMem impairment. Further, the OM $n$-back (visual-spatial sketchpad) and digit span forwards (phonological loop) and backwards (central executive) had diagnostic accuracy greater than chance for identifying those with multi-subcomponent impairment. However, after repeated administrations only the OM $n$-back assessment maintained diagnostic accuracy, detecting pwMS and those with multi-subcomponent WMem impairment; interestingly. As discussed previously, the lack of diagnostic accuracy of other measures upon repeated administration is likely due to their susceptibility to practice effects. These findings are particularly important from both a patient management and a research perspective, where serial assessments are required to ensure optimal patient management and develop targeted interventions. Consequently, the OM $n$-back might offer a more suitable method as a repeatable diagnostic measure of WMem impairment in early RRMS.

Limitations

Firstly, uneven numbers of early CDMS and CIS patients were used affecting the interpretation of subgroup differences. Secondly, it needs to be acknowledged that other WMem subcomponents, namely those ubiquitous subcomponents (central executive and episodic buffer) are likely to be involved in measures of single WMem subcomponents. Thus impairments attributed to an individual subcomponent may, in some individuals, reflect impairments in these other functions. Future studies using MRI to determine brain regions activated during task performance would allow further demarcation of WMem subcomponents impairments in early RRMS.

Concluding remarks

WMem impairments are a frequent symptom of early RRMS manifesting primarily within the visual spatial sketchpad and episodic buffer. While single WMem subcomponent impairments were the most common, a significant proportion of pwMS presented with impairments within two or more WMem subcomponents, a benchmark which proved more accurate for distinguishing WMem impaired pwMS from unimpaired individuals and healthy controls. Importantly, most WMem subcomponents did not progress within the two year study period, with deterioration only evident on visual-spatial WMem processing speed. While practice effects and differences in the psychometric properties of the measures may be a mitigating factor in this finding, it is also reasonable to conclude that WMem impairments in general progress gradually in early RRMS, and are only evident when measured concurrently with processing speed, using more sensitive methodologies. These findings provide important information for the designing of targeted intervention strategies, and the selection of tests both for screening of impairment in early RRMS and as endpoints in clinical trials. 


\section{Declarations}

Funding and/or Conflicts of interest/Competing interest

Authors Clough, Bartholomew and White have no relevant financial or non-financial interests to disclose.

Author Fielding has received funding from Biogen and Sanofi Genzyme for research related to this submission

Consent to participate

Informed consent was obtained from all individual participants included in the study

Consent to publish

All individuals consented to having their data published in this article

\section{References}

1 Brochet, B. \& Ruet, A. Cognitive Impairment in Multiple Sclerosis With Regards to Disease Duration and Clinical Phenotypes. Frontiers in neurology 10, doi:10.3389/fneur.2019.00261 (2019).

2 Fuso, S. F., Callegaro, D., Pompeia, S. \& Bueno, O. F. Working memory impairment in multiple sclerosis relapsing-remitting patients with episodic memory deficits. Arq Neuropsiquiatr 68, 205-211 (2010).

3 DeLuca, J., Barbieri-Berger, S. \& Johnson, S. K. The nature of memory impairments in multiple sclerosis: acquisition versus retrieval. J Clin Exp Neuropsychol 16, 183-189, doi:10.1080/01688639408402629 (1994).

4 DeLuca, J., Gaudino, E. A., Diamond, B. J., Christodoulou, C. \& Engel, R. A. Acquisition and storage deficits in multiple sclerosis. J Clin Exp Neuropsyc 20, 376390 (1998).

5 Ackerman, P. L., Beier, M. E. \& Boyle, M. O. Working memory and intelligence: the same or different constructs? Psychol Bull 131, 30-60, doi:10.1037/00332909.131.1.30 (2005).

6 Schwaighofer, M., Fischer, F. \& Bühner, M. Does Working Memory Training Transfer? A Meta-Analysis Including Training Conditions as Moderators.

Educational Psychologist 50, 138-166, doi:10.1080/00461520.2015.1036274 (2015).

7 DeLuca, J., Chiaravalloti, N. D. \& Sandroff, B. M. Treatment and management of cognitive dysfunction in patients with multiple sclerosis. Nat Rev Neuro/ 16, 319-332, doi:10.1038/s41582-020-0355-1 (2020).

8 Schoonheim, M. M., Geurts, J. J. \& Barkhof, F. The limits of functional reorganization in multiple sclerosis. Neurology 74, 1246-1247, doi:10.1212/WNL.0b013e3181db9957 (2010).

9 Baddeley, A. D. \& Hitch, G. in Psychology of Learning and Motivation Vol. 8 (ed Gordon H. Bower) 47-89 (Academic Press, 1974).

10 Baddeley, A. The episodic buffer: a new component of working memory? Trends Cogn Sci 4, 417-423, doi:https://doi.org/10.1016/S1364-6613(00)01538-2 (2000).

11 D'Esposito, M. \& Postle, B. R. The cognitive neuroscience of working memory. Annu Rev Psycho/ 66, 115-142, doi:10.1146/annurev-psych-010814-015031 (2015).

12 Cowan, N. in Progress in Brain Research Vol. 169 (eds Wayne S. Sossin, Jean-Claude Lacaille, Vincent F. Castellucci, \& Sylvie Belleville) $323-338$ (Elsevier, 2008).

13 Chai, W. J., Abd Hamid, A. I. \& Abdullah, J. M. Working Memory From the Psychological and Neurosciences Perspectives: A Review. Frontiers in psychology 9, doi:10.3389/fpsyg.2018.00401 (2018).

14 Blasiman, R. N. \& Was, C. A. Why Is Working Memory Performance Unstable? A Review of 21 Factors. Eur J Psycho/ 14, 188-231, doi:10.5964/ejop.v14i1.1472 (2018).

15 Ruano, L. et al. Age and disability drive cognitive impairment in multiple sclerosis across disease subtypes. Mult Scler 23, 1258-1267, doi:10.1177/1352458516674367 (2017).

16 Fuso, S. F., Callegaro, D., Pompéia, S. \& Bueno, O. F. Working memory impairment in multiple sclerosis relapsing-remitting patients with episodic memory deficits. Arq Neuropsiquiatr 68, 205-211, doi:10.1590/s0004-282x2010000200010 (2010).

17 Grzegorski, T. \& Losy, J. Cognitive impairment in multiple sclerosis - a review of current knowledge and recent research. Reviews in the Neurosciences $\mathbf{2 8 ,}$ 845-860, doi:doi:10.1515/revneuro-2017-0011 (2017). 
18 Kouvatsou, Z., Masoura, E., Kiosseoglou, G. \& Kimiskidis, V. K. Working memory profiles of patients with multiple sclerosis: Where does the impairment lie? J Clin Exp Neuropsyc 41, 832-844, doi:10.1080/13803395.2019.1626805 (2019).

19 Sumowski, J. F. et al. Cognition in multiple sclerosis: State of the field and priorities for the future. Neurology $90,278-288$, doi:10.1212/WNL.0000000000004977 (2018).

20 Clough, M. et al. Ocular motor measures of cognitive dysfunction in multiple sclerosis Il: working memory. J Neuro/ 262, 1138-1147, doi:10.1007/s00415015-7644-4 (2015).

21 Panou, T., Mastorodemos, V., Papadaki, E., Simos, P. G. \& Plaitakis, A. Early signs of memory impairment among multiple sclerosis patients with clinically isolated syndrome. Behav Neurol 25, 311-326, doi:10.3233/ben-2012-110201 (2012).

22 Reuter, F. et al. Cognitive impairment at the onset of multiple sclerosis: relationship to lesion location. Multiple Sclerosis Journal 17, 755-758, doi:10.1177/1352458511398265 (2011).

23 Viterbo, R. G., laffaldano, P. \& Trojano, M. Verbal fluency deficits in clinically isolated syndrome suggestive of multiple sclerosis. J Neurol Sci 330, 56-60, doi:10.1016/j.jns.2013.04.004 (2013).

24 Nobre, A. d. P. et al. Tasks for assessment of the episodic buffer: a systematic review. Psychology \& Neuroscience 6, 331-343 (2013).

25 Swanson, H. L., Mink, J. \& Bocian, K. M. Cognitive processing deficits in poor readers with symptoms of reading disabilities and ADHD: More alike than different? Journal of Educational Psychology 91, 321-333, doi:10.1037/0022-0663.91.2.321 (1999).

26 Jeter, C. B., Patei, S. S. \& Sereno, A. B. Novel n-back spatial working memory task using eye movement response. Behavior Research Methods 43, 879-887 (2011).

27 Strober, L. et al. Symbol Digit Modalities Test: A valid clinical trial endpoint for measuring cognition in multiple sclerosis. Mult Scler 25, 1781-1790, doi:10.1177/1352458518808204 (2019).

28 Jacobson, N. S. \& Truax, P. Clinical significance: a statistical approach to defining meaningful change in psychotherapy research. Journal of consulting and clinical psychology 59, 12-19, doi:10.1037//0022-006x.59.1.12 (1991).

29 Patti, F. et al. Cognitive impairment and its relation with disease measures in mildly disabled patients with relapsing-remitting multiple sclerosis: baseline results from the Cognitive Impairment in Multiple Sclerosis (COGIMUS) study. Mult Scler 15, 779-788, doi:10.1177/1352458509105544 (2009).

30 Nocentini, U. et al. Cognitive dysfunction in patients with relapsing-remitting multiple sclerosis. Mult Scler 12, 77-87, doi:10.1191/135248506ms1227oa (2006).

31 Achiron, A. et al. Modeling of cognitive impairment by disease duration in multiple sclerosis: A cross-sectional study. Plos One, doi:10.1371/journal.pone.0071058 (2013).

32 Ball, K., Pearson, D. G. \& Smith, D. T. Oculomotor involvement in spatial working memory is task-specific. Cognition 129, 439-446, doi:https://doi.org/10.1016/j.cognition.2013.08.006 (2013).

33 Belopolsky, A. V. \& Theeuwes, J. No functional role of attention-based rehearsal in maintenance of spatial working memory representations. Acta Psychol 132, 124-135, doi:https://doi.org/10.1016/j.actpsy.2009.01.002 (2009).

34 Achiron, A. et al. Cognitive patterns and progression in multiple sclerosis: construction and validation of percentile curves. Journal of Neurology, Neurosurgery \&amp; Psychiatry 76, 744-749, doi:10.1136/jnnp.2004.045518 (2005).

35 Clough, M. et al. Cognitive processing speed deficits in multiple sclerosis: Dissociating sensorial and motor processing changes from cognitive processing speed. Multiple sclerosis and related disorders 38, 101522, doi:10.1016/j.msard.2019.101522 (2020).

36 Clough, M. et al. Multiple sclerosis: Executive dysfunction, task switching and the role of attention. Mult Scler J Exp Trans/ Clin 4, 2055217318771781, doi:10.1177/2055217318771781 (2018).

37 Clough, M. et al. Ocular motor measures of cognitive dysfunction in multiple sclerosis I: inhibitory control. J Neuro/ 262, 1130-1137, doi:10.1007/s00415015-7645-3 (2015).

38 Fielding, J., Kilpatrick, T., Millist, L., Clough, M. \& White, O. Longitudinal assessment of antisaccades in patients with multiple sclerosis. PLoS One 7, e30475, doi:10.1371/journal.pone.0030475 (2012).

39 Gajamange, S. et al. Functional correlates of cognitive dysfunction in clinically isolated syndromes. PLoS One 14, e0219590, doi:10.1371/journal.pone.0219590 (2019).

40 Christodoulou, C. et al. Cognitive performance and MR markers of cerebral injury in cognitively impaired MS patients. Neurology 60, 1793-1798, doi:10.1212/01.wnl.0000072264.75989.b8 (2003). 
41 Lazeron, R. H., de Sonneville, L. M., Scheltens, P., Polman, C. H. \& Barkhof, F. Cognitive slowing in multiple sclerosis is strongly associated with brain volume reduction. Mult Scler 12, 760-768 (2006).

42 Huijbregts, S. C. J., Kalkers, N. F., de Sonneville, L. M. J., de Groot, V. \& Polman, C. H. Cognitive impairment and decline in different MS subtypes. J Neurol Sci 245, 187-194, doi:10.1016/j.jns.2005.07.018 (2006).

43 López-Góngora, M., Querol, L. \& Escartín, A. A one-year follow-up study of the Symbol Digit Modalities Test (SDMT) and the Paced Auditory Serial Addition Test (PASAT) in relapsing-remitting multiple sclerosis: an appraisal of comparative longitudinal sensitivity. Bmc Neuro/ 15, 40, doi:10.1186/s12883-015-02962 (2015).

44 Pavisian, B., Patel, V. P. \& Feinstein, A. Cognitive mediated eye movements during the SDMT reveal the challenges with processing speed faced by people with MS. Bmc Neurol 19, 340, doi:10.1186/s12883-019-1543-8 (2019).

45 Patel, V. P., Walker, L. A. S. \& Feinstein, A. Deconstructing the symbol digit modalities test in multiple sclerosis: The role of memory. Multiple sclerosis and related disorders 17, 184-189, doi:10.1016/j.msard.2017.08.006 (2017).

46 Camp, S. J. et al. A longitudinal study of cognition in primary progressive multiple sclerosis. Brain 128, 2891-2898, doi:10.1093/brain/awh602 (2005).

47 Roy, S. et al. Stable neuropsychiatric status in multiple sclerosis: a 3-year study. Mult Scler 22, 569-574, doi:10.1177/1352458515597570 (2016).

48 Fuchs, T. A. et al. Trait Conscientiousness predicts rate of longitudinal SDMT decline in multiple sclerosis. Mult Scler 26, 245-252,

doi:10.1177/1352458518820272 (2020).

49 McCaffrey, R. J. \& Westervelt, H. J. Issues associated with repeated neuropsychological assessments. Neuropsychol Rev 5, 203-221, doi:10.1007/BF02214762 (1995).

50 Jonides, J. How does practice makes perfect? Nat Neurosci 7, 10-11, doi:10.1038/nn0104-10 (2004).

51 Alioto, A. G. et al. Long-term test-retest reliability of the California Verbal Learning Test - second edition. The Clinical neuropsychologist 31, 1449-1458, doi:10.1080/13854046.2017.1310300 (2017).

52 Roar, M., Illes, Z. \& Sejbaek, T. Practice effect in Symbol Digit Modalities Test in multiple sclerosis patients treated with natalizumab. Multiple sclerosis and related disorders 10, 116-122, doi:10.1016/j.msard.2016.09.009 (2016).

53 Cowan, N. The magical number 4 in short-term memory: A reconsideration of mental storage capacity. Behavioral and Brain Sciences 24, 87-114, doi:10.1017/S0140525X01003922 (2001).

54 Delis, D. C., Freeland, J., Kramer, J. H. \& Kaplan, E. Integrating clinical assessment with cognitive neuroscience: Construct validation of the California Verbal Learning Test. Journal of consulting and clinical psychology 56, 123-130, doi:10.1037/0022-006X.56.1.123 (1988).

55 Mathy, F., Chekaf, M. \& Cowan, N. Simple and Complex Working Memory Tasks Allow Similar Benefits of Information Compression. J Cogn 1, 31-31, doi:10.5334/joc.31 (2018).

56 Lizak, N. et al. Impairment of Smooth Pursuit as a Marker of Early Multiple Sclerosis. Frontiers in neurology 7, 206, doi:10.3389/fneur.2016.00206 (2016).

57 Ternes, A. M., Clough, M., Foletta, P., White, O. \& Fielding, J. Characterization of inhibitory failure in Multiple Sclerosis: Evidence of impaired conflict resolution. J Clin Exp Neuropsychol 41, 320-329, doi:10.1080/13803395.2018.1552756 (2019).

58 Blekher, T. et al. Test-Retest Reliability of Saccadic Measures in Subjects at Risk for Huntington Disease. Invest Ophth Vis Sci 50, 5707-5711, doi:10.1167/iovs.09-3538 (2009).

59 Gooding, D. C., Mohapatra, L. \& Shea, H. B. Temporal stability of saccadic task performance in schizophrenia and bipolar patients. Psychol Med 34, 921932, doi:10.1017/S003329170300165X (2004).

60 Klein, C. \& Berg, P. Four-weeks test-retest stability of the saccadic CNV, two measures of saccade task performance and selected neuropsychological tests. International Journal of Psychophysiology 41, 219-219 (2001).

\section{Figures}


CVLT

(episodic buffer)

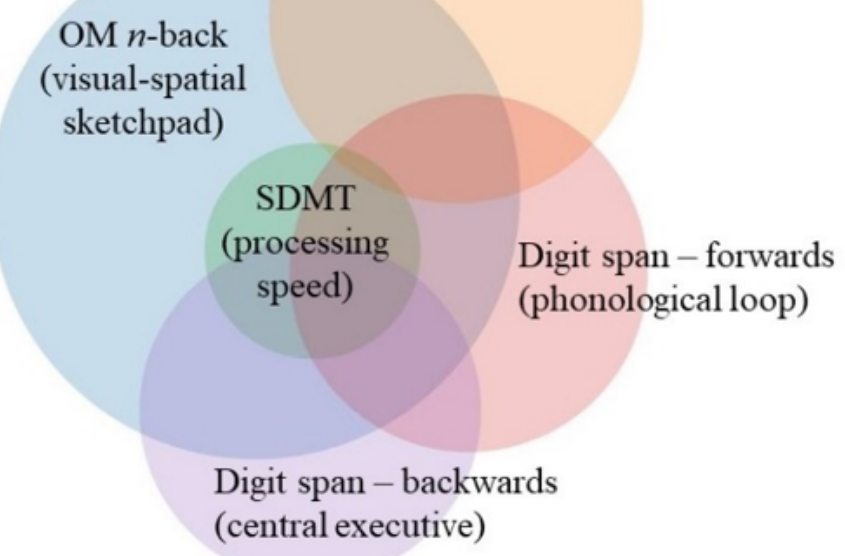

Figure 1

Proportion of pwMS impaired on each WMem measure at baseline. Size of circles indicates proportion of pwMS impaired. Degree of overlap between measures (darker areas) represents the proportion of pwMS who were impaired on more than one WMem subcomponent. Regions: OM n-back (64.86\%), SDMT (10.81\%), Digit span (45.95\%), CVLT (32.43\%). Overlap regions: OM n-back*SDMT (10.81), OM n-back*digit span (27.03\%), OM n-back*CVLT (13.51\%), Digit span*SDMT (0\%), Digit span*CVLT (5.41\%), OM n-backSDMT*Digit span (5.41\%), OM n-back*CVLT*Digit span (2.7\%). Percentages expressed as proportion of total patients impaired: $n=37$

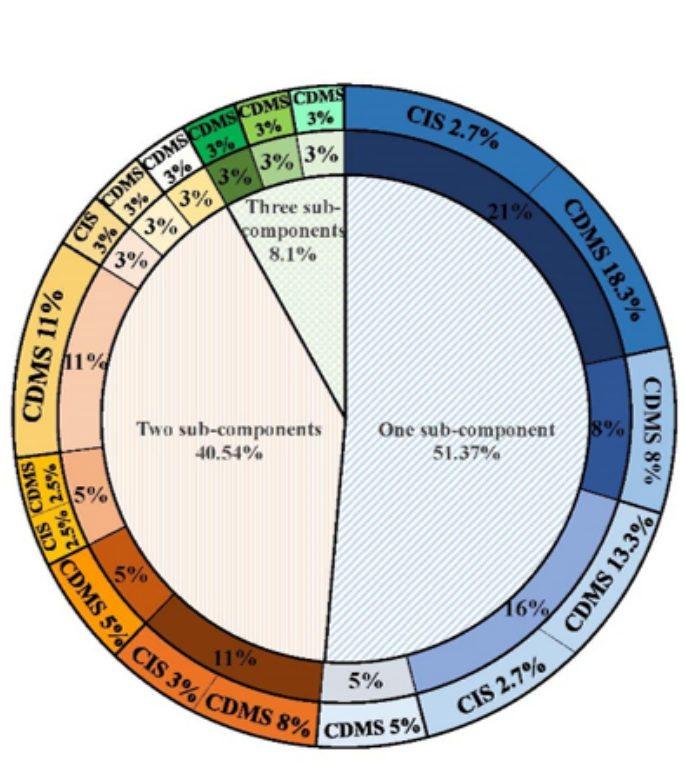

- Visual spatial sketchpad

aPhonological loop

aEpisodic buffer

$\square$ Central executive

- Visual spatial sketchpad + Episodic buffer

aisual spatial sketchpad + Cognitive processing speed

$\square$ Visual spatial sketchpad + Phonological loop

$\square$ Visual spatial sketchpad + Central executive

-Episodic buffer + Phonological loop

$\square$ Phonological loop + Cognitive processing speed

$\square$ Central cxecutive + Cognitive processing specd

-Visual spatial sketchpad + phonological loop + Cognitive processing specd

$\square$ Visual spatial sketchpad + Episodic buffer + Phonological loop

$\square$ Visual spatial skctchpad + Phonological loop + Central executive

\section{Figure 2}

Frequency of WMem impairment within each subcomponent. Frequencies are represented in regards to MS subgroups (CIS, CDMS) and number of WMem subtypes impaired. 